\title{
Wave propagation in strongly scattered random elastic media: Energy equilibration and crossover from ballistic to diffusive behavior
}

\author{
Ying Wu, Yun Lai, Yanyi Wan, and Zhao-Qing Zhang \\ Department of Physics, Hong Kong University of Science and Technology, Clear Water Bay, Kowloon, Hong Kong
}

(Received 11 December 2007; revised manuscript received 10 February 2008; published 17 March 2008)

\begin{abstract}
We studied the energy equilibration process of elastic wave propagation through a strong-scattering random medium via multiple-scattering theory and the radiative transfer equation. The equilibration of the shear and compressional energy densities due to the mode conversions is clearly observed in both calculations, although the ratio of the shear energy density to the compressional energy density obtained from the multiple-scattering theory is higher than that obtained from the radiative transfer equation, which has the value predicted by the principle of the equipartition of wave modes. The discrepancy is due to the presence of a negative interference energy inside the sample. This is in contrast to the common belief that the interference energy density of a weak-scattering random medium always averages to zero inside the medium except near its boundaries. We also showed that the negative interference energy is concentrated near the boundary of each scatterer and, therefore, cannot be averaged to zero. In addition, we studied various distribution functions of the transmitted waves in thin samples before the establishment of the energy equilibration. We found that these distribution functions are described well by a random-phasor-sum model and they exhibit crossover behavior from ballistic to diffusive transport.
\end{abstract}

DOI: 10.1103/PhysRevB.77.125125

PACS number(s): 46.40.Cd, 42.25.Dd, 62.30.+d

\section{INTRODUCTION}

In the past two decades, great progress has been made in the study of the propagation of classical waves in random media. ${ }^{1}$ One important subject is the multiple scattering of elastic waves in complex solid media, particularly in relation to high-frequency seismic wave propagation ${ }^{2}$ and the ultrasonic characterization of polycrystalline metals. ${ }^{3,4}$ Unlike other classical waves, there are two types of elastic waves propagating in solids, i.e., shear (S) waves and compressional (P) waves. This leads to the principle of equipartition in the diffusive regime, which states that the ratio of S-wave energy to P-wave energy converges to a universal number independent of the fluctuations that cause the scattering. ${ }^{4-8}$ This number is believed to be the ratio of density of states of the two modes in an unbounded medium ${ }^{5}$ or equivalently $\left(c_{p} / c_{s}\right)^{d},{ }^{9}$ where $d$ is the dimensionality of the medium and $c_{p}$ and $c_{s}$ are, respectively, the compressional and shear wave speeds. The equipartition of elastic waves has been theoretically studied by using the radiative transfer equation (RTE) and the diffusion equation. ${ }^{6-8}$ Results showed that the energy equilibration is reached inside a random medium after a sufficient number of multiple scatterings, and the ratio is independent of the magnitude or polarization of the source. Recently, the equipartition of seismic coda waves created by earthquakes was observed by Hennino et al. ${ }^{10}$ Interestingly, in addition to the $\mathrm{S}$-wave and $\mathrm{P}$-wave energies, they also observed the existence of a negative average interference energy $\left\langle E_{i}\right\rangle$ at the earth's free surface due to boundary-induced mode conversions. They also predicted that the nonvanishing $E_{i}$ appears only within a distance of a few shear wavelengths from the surface.

Present theoretical approaches are generally based on the RTE. However, this approach considers only the energy densities of $\mathrm{S}$ and $\mathrm{P}$ waves and assumes that the interference energy is averaged to zero. This is true in the weak-scattering limit when the average distance between two scatterers is large compared to the wavelengths, and thus the near-field effects can be ignored. However, when the average distance between two scatterers becomes comparable to the wavelengths, the near-field effects cannot be ignored and $E_{i}$ may not be averaged to zero inside the medium. To the best of our knowledge, such near-field effects have not been reported. Thus, investigating how these near-field effects affect the statistical properties of elastic wave propagation in strongscattering random media and determining to what extent the RTE still applies are both interesting issues.

For these purposes, we studied elastic wave propagation through a strong-scattering, two-dimensional random elastic medium. We chose the random medium and the frequency of the waves such that the average separation of two scatterers was comparable to the wavelengths of both $\mathrm{S}$ waves and $\mathrm{P}$ waves. We utilized two theoretical approaches in this study. First, we used the multiple-scattering theory (MST) method in conjunction with a sparse-matrix canonical grid (SMCG) method $^{11}$ to run large-scale numerical simulations of elastic wave propagation. The SMCG method greatly enhances the efficiency of the MST method, particularly with large-sized samples containing large number of scatterers. Through this first-principles approach, we calculated the shear energy density $E_{s}$, the compressional energy density $E_{p}$, and the inference energy density $E_{i}$ inside the samples. Second, we solved the RTE by using realistic physical parameters determined in the first approach. Since the interference energy density is assumed to be averaged to zero, only the shear energy density, $E_{s}^{R T E}$ and the compressional energy density $E_{p}^{R T E}$ can be calculated inside the samples. Both $\mathrm{S}$ and $\mathrm{P}$ wave incidences were considered in both approaches.

We present three main results. First, in contrast to the common belief, our MST results show a negative $\left\langle E_{i}\right\rangle$ across the sample, which does not average to zero in a random environment. To understand the origin of the negative $\left\langle E_{i}\right\rangle$, 
we also studied various energy density distributions resulting from a plane-wave scattering by a single scatterer. We found that the negative $\left\langle E_{i}\right\rangle$ is mainly concentrated near the boundary of the scatterers and is always accompanied by an enhancement of the shear energy density by a similar amount. Since such near-field effects occur near the boundary, they cannot be averaged to zero. Their existence also causes the RTE to be inaccurate in a strong-scattering medium. Second, the results of both MST calculations and RTE show clearly an energy equilibration process, i.e., the ratio of the shear energy density to the compressional energy density approaches to a constant value inside the sample, which is independent of the source polarization. In the case of RTE, the equilibrated value of $E_{s}^{R T E} / E_{p}^{R T E}$ agrees well with the principle of equipartition proposed in Ref. 5, i.e., $\left(c_{p} / c_{s}\right)^{2}$ in two dimensions. However, the multiple-scattering results give a higher equilibrated ratio of $\left\langle E_{s}\right\rangle /\left\langle E_{p}\right\rangle$ than $\left(c_{p} / c_{s}\right)^{2}$. This is due to the existence of a negative $\left\langle E_{i}\right\rangle$ across the sample. It is interesting that the spatial distributions of $\left(\left\langle E_{s}\right\rangle+\left\langle E_{i}\right\rangle\right)$ and $\left\langle E_{p}\right\rangle$ agree well with the distributions of the shear and compressional energy densities obtained by the RTE, i.e., $E_{s}^{R T E}$ and $E_{p}^{R T E}$. Thus, the ratio of $\left(\left\langle E_{s}\right\rangle+\left\langle E_{i}\right\rangle\right)$ to $\left\langle E_{p}\right\rangle$ approaches the expected ratio of $\left(c_{p} / c_{s}\right)^{2}$. This is a result of the cancellation between the enhancement of $\left\langle E_{s}\right\rangle$ and the negative $\left\langle E_{i}\right\rangle$ near the boundary of the scatterer. Finally, by using the MST method, we have also studied the statistics of transmitted fields in thin samples before the establishment of energy equilibration. By separating the transmitted field into a coherent part and a diffusive part and studying the amplitude and phase distributions of both parts, we also observed the crossover from ballistic to diffusive wave behavior through an analysis based on a random-phasor-sum (RPS) model. This model considers the random transmitted field as a sum of a circular random phasor and a constant, and it was proposed to explain the statistics of transmitted fields obtained in microwave experiments through random waveguides. ${ }^{12}$ This model was later applied to transmitted fields through randomly corrugated waveguides ${ }^{13}$ and reflected fields in disordered photonic crystals. ${ }^{14}$

This paper is organized as follows: In Sec. II, we introduce our system and simulation method. The RTE is presented in Sec. III, where we first discuss all the scattering mean free paths for our system and then the integral solution to the RTE for elastic waves in two dimensions. The results obtained from both the MST and the RTE are presented and discussed in Sec. IV. The field distributions in thin samples and the associated crossover behavior from ballistic to diffusive are presented in Sec. V. Conclusions are presented in Sec. VI.

\section{SYSTEM AND SIMULATION METHOD}

We obtained our random medium by randomizing a twodimensional (2D) elastic phononic crystal consisting of a square array of epoxy cylinders with a radius of $r_{s}$ and a set of elastic parameters $\left(\rho_{s c a}, \lambda_{s c a}, \mu_{s c a}\right)$, embedded in an iron host with another set of elastic parameters $\left(\rho_{0}, \lambda_{0}, \mu_{0}\right)$, where $\rho$ denotes the mass density and $\lambda$ and $\mu$ represent the Lamé

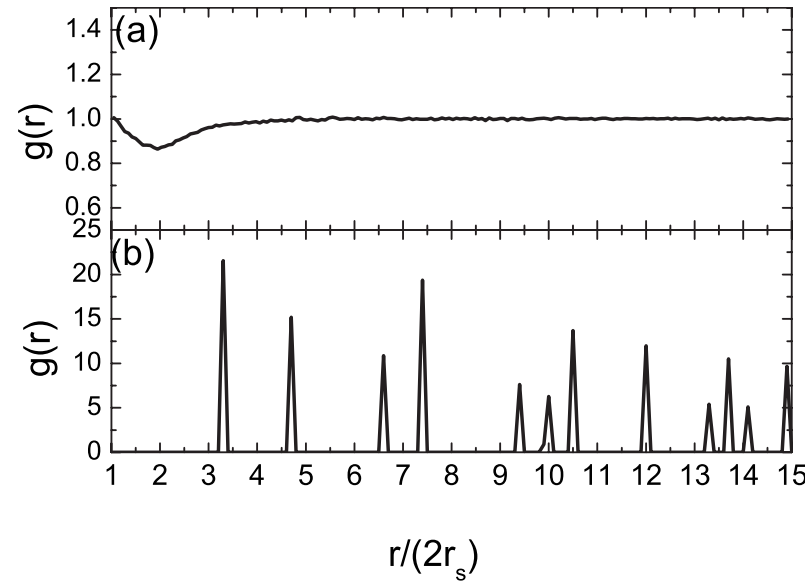

FIG. 1. The radial distribution function $g(r)$ as function of distance away from the center of the cylinder. (a) For random systems. (b) For a $2 \mathrm{D}$ phononic crystal in a square lattice.

constants. The lattice constant of the phononic crystal is $a$.

To randomize the phononic crystal, we moved the position of each cylinder randomly within a distance of $0.5 a$. If two cylinders overlapped, the move was forbidden. The procedure was repeated 1000 times to randomize the positions of the scatterers completely. We generated an ensemble of 200 configurations for our study.

To ensure that our samples are completely randomized, we calculated the radial distribution function (RDF), $g(r)$, according to Eq. (10) of Ref. 15. For any given random configuration of a square sample of size $40 a$, we arbitrarily chose one cylinder located near the center of the sample as the coordinate origin. We calculated the RDF by sweeping circular rings of constant width $d r$ radially outward from the origin to $R$, the outer radius of the largest ring. The largest ring should not touch the boundary of the sample. We counted the number of cylinders, $d N(r)$, inside an annular ring at distance $r$ and calculated RDF by using $g(r)$ $=(d N / d V) /(N / V),{ }^{15}$ where $d V$ is the area of the annular ring, $N$ is the total numbers of cylinders inside all the rings, and $V$ represents the total area occupied by all the rings. In our case, the ratio of $N / V$ is $1 / a^{2}$ on average.

The averaged results of $g(r)$ is shown by a solid curve in Fig. 1(a). For comparison, we also show the RDF of the ordered phononic crystal in Fig. 1(b). The absence of any peaks in Fig. 1(a) demonstrates that the random systems generated here are completely random without any residual periodicity. In fact, our result is very similar to Fig. 3(a) of the Ref. 15, where the RDF was obtained from a random distribution of cylinders in a 2D sample at a small filling factor.

The samples used in our study had a width of $W=50 a$ and different thicknesses. For a given thickness $L$, the total number of cylinders in the sample is $W L / a^{2}=50 L / a$. The whole sample was embedded in the material which is the same as the sample's matrix, i.e., iron in this case, so that the wave could leak out freely without being reflected at the sample's boundary. The source was a plane wave passing through an open slit located about two lattice constants in front of the sample. The width of the slit was $40 a$, which was about $20 \%$ smaller than that of the sample to avoid the diffraction caused by the sample's edges. 
The displacement field $\mathbf{u}$ inside the $x-y$ plane satisfies the elastic wave equation: ${ }^{16}$

$$
\begin{aligned}
\rho \frac{\partial^{2} u_{i}}{\partial t^{2}}= & \nabla \cdot\left(\rho c_{t}^{2} \nabla u_{i}\right)+\nabla \cdot\left(\rho c_{l}^{2} \frac{\partial \mathbf{u}}{\partial x_{i}}\right) \\
& +\frac{\partial}{\partial x_{i}}\left[\left(\rho c_{l}^{2}-2 \rho c_{t}^{2}\right) \nabla \cdot \mathbf{u}\right], \quad i=x, y,
\end{aligned}
$$

where $c_{p}=\sqrt{(\lambda+2 \mu) / \rho}$ and $c_{s}=\sqrt{\mu / \rho}$ denote the compressional and shear wave speeds, respectively. In general, the displacement field $\mathbf{u}$ can be decoupled into compressional and shear parts, i.e., $\mathbf{u}=\mathbf{u}_{p}+\mathbf{u}_{s}=\nabla \phi_{p}+\nabla \times\left(\phi_{s} \hat{e}_{z}\right)$, where $\phi_{p}$ and $\phi_{s}$ are the scalar potential functions. In terms of these two scalar potentials, Eq. (1) can be rewritten, in the frequency domain, as two Helmholtz equations:

$$
\left(\nabla^{2}+k_{p}^{2}\right) \phi_{p}=0, \quad\left(\nabla^{2}+k_{s}^{2}\right) \phi_{s}=0,
$$

where $k_{p}=\omega / c_{p}$ and $k_{s}=\omega / c_{s}$ are the compressional and shear wave vectors and $\omega$ is the angular frequency. The general solution to Eq. (2) can be written as $\phi_{\alpha}(s c a)$ $=\Sigma_{m} a_{\alpha m}(s c a) J_{m}\left(k_{\alpha s c a} r\right) e^{i m \theta}(\alpha=s, p)$ in the cylinder, where $k_{\alpha s c a}$ are the wave vectors in the cylinders, and $\phi_{\alpha}(0)$ $=\Sigma_{m}\left[a_{\alpha m}(0) J_{m}\left(k_{\alpha 0} r\right)+b_{\alpha m}(0) H_{m}^{(1)}\left(k_{\alpha 0} r\right)\right] e^{i m \theta}(\alpha=s, p)$ in the matrix, where $k_{\alpha 0}$ are the wave vectors in the matrix. Here, $J_{m}$ and $H_{m}^{(1)}$ are Bessel functions and Hankel functions of the first kind, respectively. The coefficients of the Bessel and Hankel functions can be determined by the boundary conditions, which are the continuities of the radial and tangential components of the displacement field, i.e., $u_{r}$ and $u_{\theta}$, and the continuities of the stresses, $\sigma_{r r}$ and $\sigma_{r \theta}$, on the surface of each cylinder. The stresses are defined as

$$
\begin{gathered}
\sigma_{r r}=(\lambda+2 \mu) \frac{\partial u_{r}}{\partial r}+\lambda\left(\frac{1}{r} \frac{\partial u_{\theta}}{\partial \theta}+\frac{u_{r}}{r}\right), \\
\sigma_{r \theta}=\mu\left(\frac{1}{r} \frac{\partial u_{r}}{\partial \theta}+\frac{\partial u_{\theta}}{\partial r}-\frac{u_{\theta}}{r}\right) .
\end{gathered}
$$

The continuities on the surface of a cylinder relate $b_{s m}(0)$ and $b_{p m}(0)$ to $a_{s m}(0)$ and $a_{p m}(0)$ through $b_{s m}(0)=D_{m}^{s s} a_{s m}(0)$ $+D_{m}^{s p} a_{p m}(0) \quad$ and $\quad b_{p m}(0)=D_{m}^{p s} a_{s m}(0)+D_{m}^{p p} a_{p m}(0)$, where $D_{m}^{\alpha \beta}(\alpha, \beta=s, p)$ are the Mie-like scattering coefficients determined by the boundary conditions at the interface between the cylinder and the matrix. ${ }^{17}$ Then, the scattering properties of the system can be calculated by the MST method. The MST method is a well-established numerical method and has been used to study the scattering and transmission properties of a finite-sized sample. ${ }^{18-20}$ The MST method gives rise to a set of linear equations. The number of linear equations for elastic waves is twice that for electromagnetic or acoustic waves in two dimensions, due to the existence of both compressional and transverse waves in elastic media. The operation cost of solving these equations is $O\left(\left(2 N_{m}\right)^{3}\right)$, while the memory cost is $O\left(\left(2 N_{m}\right)^{2}\right)$. Here, $N_{m}$ is the number of cylinders, $N$, multiplied by $(2 m+1) . m$ is the cutoff angular momentum quantum number used in the calculations. Thus, the calculations become extremely time consuming when the number of cylinders or the number of configurations is large.
However, the difficulty can be overcome by incorporating the so-called SMCG method into the MST method. ${ }^{11}$ The SMCG method is an efficient algorithm based on the decomposition of strong and weak interactions among cylinders. Here, the strong and weak interactions are the interactions between the near and distant cylinders, respectively. Basically, the idea is to utilize a two-dimensional uniform canonical grid that covers the sample. Every cylinder is associated with its nearest grid point. Then, the weak interactions, which consume the majority of the required CPU time and memory, can be calculated by using the grid points. With the successive use of the addition theorem, the weak interactions between two distinct cylinders are calculated indirectly from one cylinder to its associated grid point, then from the grid point to the grid point associated with the other cylinder, and finally from the second grid point to the other cylinder. This facilitates the use of fast Fourier transform, which reduces the operation cost from $O\left(\left(2 N_{m}\right)^{3}\right)$ to $O\left(\left(2 N_{m}\right) \log \left(2 N_{m}\right)\right) .{ }^{11}$ In our calculations, we have used an iterative method called the generalized minimal residue (GMRES) method ${ }^{21}$ to solve the coupled linear equations given by MST. The GMRES method is very robust and accurate even for dense matrices, and it reduces the memory cost from $O\left(\left(2 N_{m}\right)^{2}\right)$ to $O\left(2 N_{m}\right)$. We have also checked the convergence of our results by increasing the cutoff angular momentum quantum number $m$ and decreasing normwise backward error in GMRES to perform our calculations. We finally adopted $m=10$ and backward error equals to $10^{-6}$, while the result showed that the convergence has already been reached when $m=8$ and the normwise backward error in GMRES equals to $10^{-5}$.

Once we obtained the displacement fields, it is straightforward to calculate the energy densities by using the following formula: ${ }^{22}$

$$
\begin{aligned}
E= & E_{s}+E_{p}+E_{i}=\frac{\mu}{2}(\nabla \times \mathbf{u})^{2}+\left(\frac{\lambda}{2}+\mu\right)(\nabla \cdot \mathbf{u})^{2} \\
& +\mu \sum_{i \neq j}\left(\frac{\partial u_{i}}{\partial x_{j}} \frac{\partial u_{j}}{\partial x_{i}}-\frac{\partial u_{i}}{\partial x_{i}} \frac{\partial u_{j}}{\partial x_{j}}\right),
\end{aligned}
$$

where $E_{s}$ and $E_{p}$ are the shear and compressional energy densities, respectively. The term $E_{i}$, which involves cross terms, is the interference energy density caused by the interference effects of the $\mathrm{S}$ and $\mathrm{P}$ waves. This term is expected to average to zero inside a random medium after configurational averaging. However, we will show later that $E_{i}$ is peaked near the boundary of the scatterer and, therefore, cannot be averaged to zero in the case of the strong-scattering media considered here.

As mentioned previously, our system consists of randomly placed epoxy cylinders in an iron host. The epoxy cylinder has a radius of $r_{s}=0.15 a$. The mass densities of iron and epoxy are $\rho_{0}=7780 \mathrm{~kg} / \mathrm{m}^{3}$ and $\rho_{s}=1142 \mathrm{~kg} / \mathrm{m}^{3}$, respectively. The compressional wave speeds inside the iron and epoxy are $c_{p 0}=5825.2 \mathrm{~m} / \mathrm{s}$ and $c_{p s}=2569.5 \mathrm{~m} / \mathrm{s}$, respectively. The shear wave speeds are $c_{s 0}=3226.7 \mathrm{~m} / \mathrm{s}$ for iron and $c_{s s}=1138.4 \mathrm{~m} / \mathrm{s}$ for epoxy. ${ }^{23}$ 

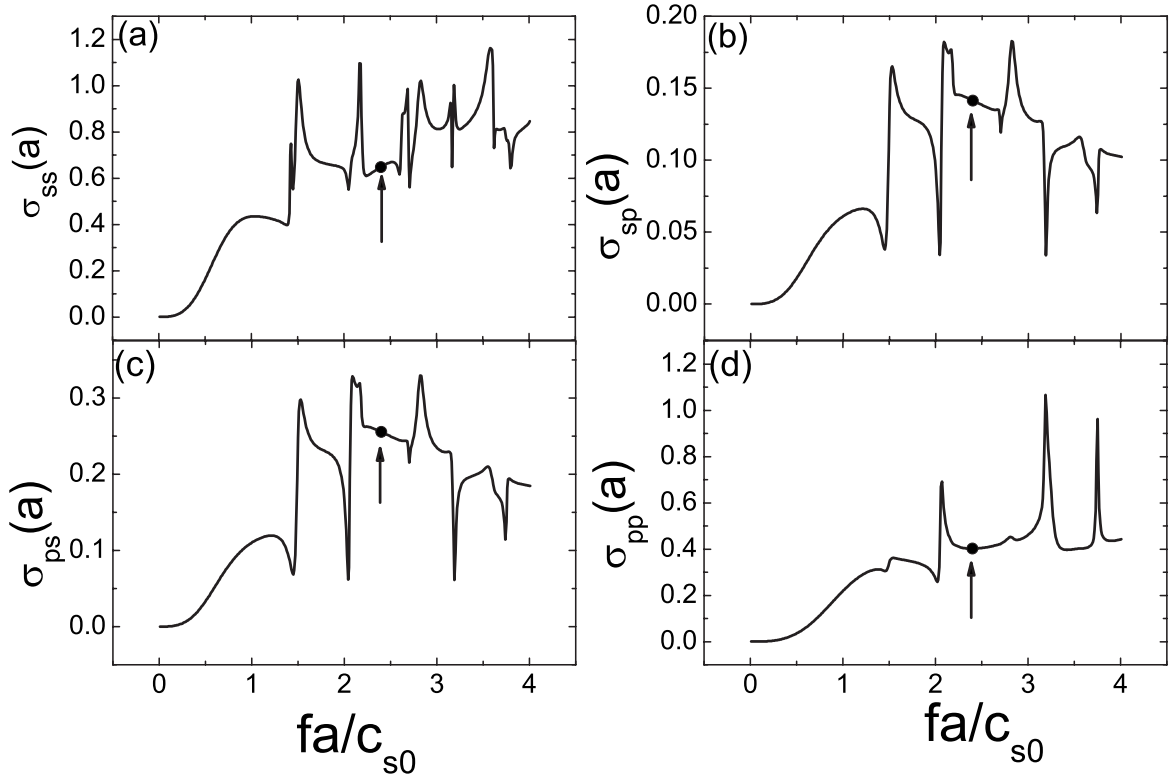

FIG. 2. The total scattering cross section for a epoxy cylinder embedded in an iron host vs the dimensionless frequency. (a) From S-to-S wave; (b) from S-to-P wave; (c) from P-to-S wave; (d) from P-to-P wave.

\section{THE RADIATIVE TRANSFER EQUATION}

Before we present the RTE, we first discuss the relevant single-scattering properties of our system, i.e., various scattering cross sections and scattering mean free paths. These quantities are required in solving the RTE. The scattering mean free paths can be obtained from the scattering properties of each individual cylinder. First, let us consider the case of shear wave incidence. For an S-polarized plane-wave incident along the $x$ axis, the displacement field, $\mathbf{u}_{s}^{i n c}$, has the form

$$
\mathbf{u}_{s}^{i n c}(\mathbf{r})=A e^{i k_{s} x} \hat{\mathbf{y}},
$$

where $A$ represents the amplitude. The scattered wave in the far field has the form

$$
\mathbf{u}^{s c a}(\mathbf{r}) \rightarrow \hat{\mathbf{r}} \frac{f^{s p}(\theta)}{\sqrt{r}} A e^{i k_{p} r}+\hat{\theta} \frac{f^{s s}(\theta)}{\sqrt{r}} A e^{i k_{s} r},
$$

where $f^{s s}(\theta)$ and $f^{s p}(\theta)$ are the angular distributed amplitudes of the scattered shear and compressional waves, respectively. With these values, the total scattering cross sections for the $\mathrm{S}$-to-S-wave and S-to-P-wave scatterings can be obtained through the relations $\sigma_{s s}=\int_{2 \pi}\left|f^{s s}(\theta)\right|^{2} d \theta$ and $\sigma_{s p}$ $=\int_{2 \pi}\left(c_{p} / c_{s}\right)\left|f^{s p}(\theta)\right|^{2} d \theta$, respectively. The total scattering cross section for the shear wave is the sum of $\sigma_{s s}$ and $\sigma_{s p}$, i.e.,

$$
\sigma_{s}=\sigma_{s s}+\sigma_{s p}
$$

Similarly, for a P-polarized plane-wave incidence, the total scattering cross section becomes

$$
\sigma_{p}=\sigma_{p s}+\sigma_{p p},
$$

where $\sigma_{p s}=\int_{2 \pi}\left(c_{s} / c_{p}\right)\left|f^{p s}(\theta)\right|^{2} d \theta$ and $\sigma_{p p}=\int_{2 \pi}\left|f^{p p}(\theta)\right|^{2} d \theta$ are the total scattering cross sections for the P-to-S- and P-to-Pwave scatterings, respectively.

Here, we have four scattering mean free paths: $l_{s s}, l_{s p}, l_{p s}$, and $l_{p p}$, corresponding to the scattering of S-to-S-, S-to-P-, $\mathrm{P}-$ to-S- and P-to-P waves, respectively. Since the scattering mean free path is determined by the inverse of the product of the total scattering cross section and the number density of the scatterer, the corresponding scattering mean free paths are

$$
\begin{gathered}
l_{s s}=\frac{1}{n \sigma_{s s}}, \quad l_{s p}=\frac{1}{n \sigma_{s p}}, \\
l_{p s}=\frac{1}{n \sigma_{p s}}, \quad l_{p p}=\frac{1}{n \sigma_{p p}},
\end{gathered}
$$

where $n$ is the number density, which is equal to 1 in our system. Combining Eqs. (8) with Eqs. (6) and (7), we obtain the scattering mean free paths for the $\mathrm{S}$ and $\mathrm{P}$ waves as

$$
\begin{aligned}
& \frac{1}{l_{s}}=\frac{1}{l_{s s}}+\frac{1}{l_{s p}}, \\
& \frac{1}{l_{p}}=\frac{1}{l_{p s}}+\frac{1}{l_{p p}} .
\end{aligned}
$$

Physically, $l_{s}$ or $l_{p}$ gives the decay length of the coherent intensity inside the sample, which also corresponds to the phase randomization of the incident wave after a distance of $l_{s} / 2$ or $l_{p} / 2$ due to the scattering.

The single-scattering properties of our system are shown in Fig. 2, where a dimensionless frequency, $\tilde{f}=f a / c_{s 0}$, is used. In our study, we chose a particular frequency, $\tilde{f}=2.4$, indicated by the arrows in Fig. 2. The reason we chose this frequency is that it is not close to any resonances shown in Figs. 1(a)-1(d). Furthermore, at this frequency, the four total scattering cross sections are not small, i.e., $\sigma_{s s}=0.649 a, \sigma_{s p}$ $=0.141 a, \sigma_{p s}=0.255 a$, and $\sigma_{p p}=0.402 a$ and, therefore, the corresponding scattering mean free paths are not large, i.e., $l_{s s}=1.54 a, l_{s p}=7.09 a, l_{p s}=3.92 a$, and $l_{p p}=2.49 a$, which give $l_{s}=1.26 a$ and $l_{p}=1.52 a$. This makes our numerical simulations more feasible. 
An elastic RTE was derived from first principles by Weaver ${ }^{4}$ and Ryzhik et al. ${ }^{24}$ From this equation, many aspects of energy transport behavior of elastic waves in random media can be determined, such as mode conversion and equipartition, etc. However, this approach deals with weakscattering random media and, therefore, the interference energy is assumed to be averaged to zero inside an unbounded random medium. In two dimensions, the RTE takes a simple form:

$$
\begin{aligned}
\hat{s} \cdot \nabla I_{i}(\mathbf{r}, \hat{s})+\frac{I_{i}(\mathbf{r}, \hat{s})}{l_{i}}= & \frac{1}{2 \pi} \sum_{j=1,2} \int_{0}^{2 \pi} P_{i j}\left(\hat{s}^{\prime}, \hat{s}\right) \\
& \times I_{j}\left(\mathbf{r}, \hat{s}^{\prime}\right) d \hat{s}^{\prime}, \quad i=1,2,
\end{aligned}
$$

where $I_{1}=I_{p}$ and $I_{2}=I_{s}$ are the compressional and shear energy current densities, respectively. $l_{1}$ and $l_{2}$ correspond to the total scattering mean free paths of $\mathrm{P}$ and $\mathrm{S}$ waves defined previously, i.e., $l_{p}$ and $l_{s}$, respectively. $\underline{\underline{P}}\left(\hat{s}^{\prime}, \hat{s}\right)$ is the reduced Muller matrix, which governs the scattering of the intensity from direction $\hat{s}^{\prime}$ to direction $\hat{s}$. For a medium containing discrete scatterers, the matrix is related to the number density $n$ and the single scatterer differential cross sections and has the following form:

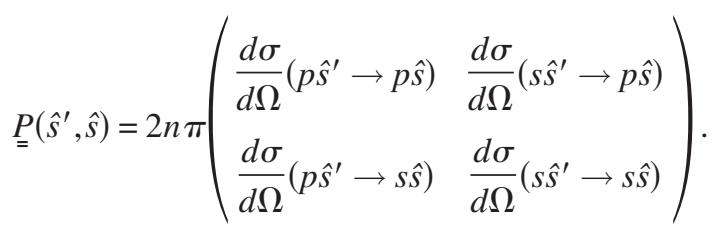

Now, we consider a plane wave normally incident on the input surface of a sample of thickness $L$ at position $x=0$. Due to the symmetry, the energy current density only depends on $x$. For simplicity, we assume the sample boundaries are nonreflecting, so we neglect internal reflections inside the sample. This leads to simple boundary conditions that the forward propagating energy current density at the input boundary equals to the original incident energy current density, and the backward propagating energy current density at the output boundary equals to zero, i.e., $I_{p, s}(x=0, \theta)$ $=I_{p 0, s 0} \delta(\theta-0)$ for $-\pi / 2<\theta<\pi / 2$ and $I_{p, s}(x=L, \theta)=0$ for $\pi / 2<\theta<3 \pi / 2$. The use of nonreflecting boundary condition in the RTE is consistent with the random systems we used for MST calculation. It is easy to show that the solution to Eq. (10) satisfies the following two integral equations:

$$
\begin{aligned}
\left(\begin{array}{l}
I_{p}(x, \theta) \\
I_{s}(x, \theta)
\end{array}\right)= & \left(\begin{array}{l}
I_{p 0} e^{-x / l_{p}} \\
I_{s 0} e^{-x / l_{s}}
\end{array}\right) \delta(\theta-0) \\
& +\int_{0}^{x} \frac{d x^{\prime}}{\cos \theta}\left(\begin{array}{cc}
e^{-\left(x-x^{\prime}\right) /\left(l_{p} \cos \theta\right)} & 0 \\
0 & e^{-\left(x-x^{\prime}\right) /\left(l_{s} \cos \theta\right)}
\end{array}\right) \\
& \times \int_{0}^{2 \pi}\left(\begin{array}{ll}
P_{p p} & P_{s p} \\
P_{p s} & P_{s s}
\end{array}\right)\left(\begin{array}{c}
I_{p}\left(x^{\prime}, \theta^{\prime}\right) \\
I_{s}\left(x^{\prime}, \theta^{\prime}\right)
\end{array}\right) d \theta^{\prime}
\end{aligned}
$$

in the forward direction, i.e., $0<|\theta|<\pi / 2$, and

$$
\begin{aligned}
\left(\begin{array}{l}
I_{p}(x, \theta) \\
I_{s}(x, \theta)
\end{array}\right)= & \int_{L}^{x} \frac{d x^{\prime}}{\cos \theta}\left(\begin{array}{cc}
e^{-\left(x-x^{\prime}\right) /\left(l_{p} \cos \theta\right)} & 0 \\
0 & e^{-\left(x-x^{\prime}\right) /\left(l_{s} \cos \theta\right)}
\end{array}\right) \\
& \times \int_{0}^{2 \pi}\left(\begin{array}{ll}
P_{p p} & P_{s p} \\
P_{p s} & P_{s s}
\end{array}\right)\left(\begin{array}{l}
I_{p}\left(x^{\prime}, \theta^{\prime}\right) \\
I_{s}\left(x^{\prime}, \theta^{\prime}\right)
\end{array}\right) d \theta^{\prime}
\end{aligned}
$$

in the backward direction, i.e., $\pi / 2<|\theta|<\pi$. Here, a matched boundary condition has been assumed.

We solve Eqs. (12) and (13) numerically and obtain the energy density, $E_{p, s}^{R T E}$, by using the relation

$$
E_{p, s}^{R T E}(x)=\int_{0}^{2 \pi} I_{p, s}(x, \hat{s}) / c_{p, s} d \hat{s} .
$$

According to the equipartition principle discussed in Ref. 5 , we expect that the ratio $E_{s}^{R T E}(x) / E_{p}^{R T E}(x)$ approaches a constant value that equals the ratio of the density of states (DOS) of the two modes when $x$ is sufficiently large. Since in $2 \mathrm{D}$, the DOS is inversely proportional to the square of the wave speed, the equilibrated ratio of $E_{s}^{R T E}(x) / E_{p}^{R T E}(x)$ is expected to be $\left(c_{p} / c_{s}\right)^{2} .9$

\section{RESULTS AND DISCUSSIONS}

Figures 3(a) and 3(b) show the results of various energy densities inside a sample with thickness $L=14 a$ for the Sand P-wave incidences, respectively. The solid, dashed, and dotted curves represent the shear $\left(E_{s}^{R T E}\right)$, compressional $\left(E_{p}^{R T E}\right)$, and total $\left(E_{\text {total }}^{R T E}\right)$ energy densities calculated using the RTE. The open circles, open squares, open up triangles, and open down triangles represent, respectively, the averaged shear $\left(\left\langle E_{s}\right\rangle\right)$, compressional $\left(\left\langle E_{p}\right\rangle\right)$, interference $\left(\left\langle E_{i}\right\rangle\right)$, and total $\left(\left\langle E_{\text {total }}\right\rangle\right)$ energy densities calculated by using the MST method. These results are obtained from an average of 200 configurations. We also normalized the results of two different calculations in a way that $\left\langle E_{p}\right\rangle$ has the same value as $E_{p}^{R T E}$ in the middle of the sample. From these two figures, it is interesting to observe that the interference energy density calculated by the MST method does not average to zero. It has a negative value across the sample, i.e., $\left\langle E_{i}\right\rangle<0$. It is also interesting to observe that the spatial distributions of both $\left\langle E_{p}\right\rangle$ and $\left\langle E_{\text {total }}\right\rangle$ agree excellently with $E_{p}^{R T E}$ and $E_{\text {total }}^{R T E}$, respectively, although the shear energy densities obtained from the two methods are different. This observation implies that the sum of the shear and interference energy densities calculated by the MST method should also agree well with the shear energy density calculated by the RTE. To check this, we plot the sum $\left\langle E_{s}\right\rangle+\left\langle E_{i}\right\rangle$ by solid circles in Figs. 3(a) and $3(\mathrm{~b})$, which indeed overlaps with the solid curves $\left(E_{s}^{R T E}\right)$ obtained from the RTE.

To examine the behavior of energy equilibration, we plot the ratio of the shear energy density to the compressional energy density in Fig. 4(a). For the case of S-wave incidence, the results obtained from the RTE and the MST are shown by a solid curve and open squares, respectively. For the case of $\mathrm{P}$-wave incidence, the corresponding results are shown by a dashed curve and open circles. It is clearly seen that both the results of the RTE and the MST clearly exhibit an energy 


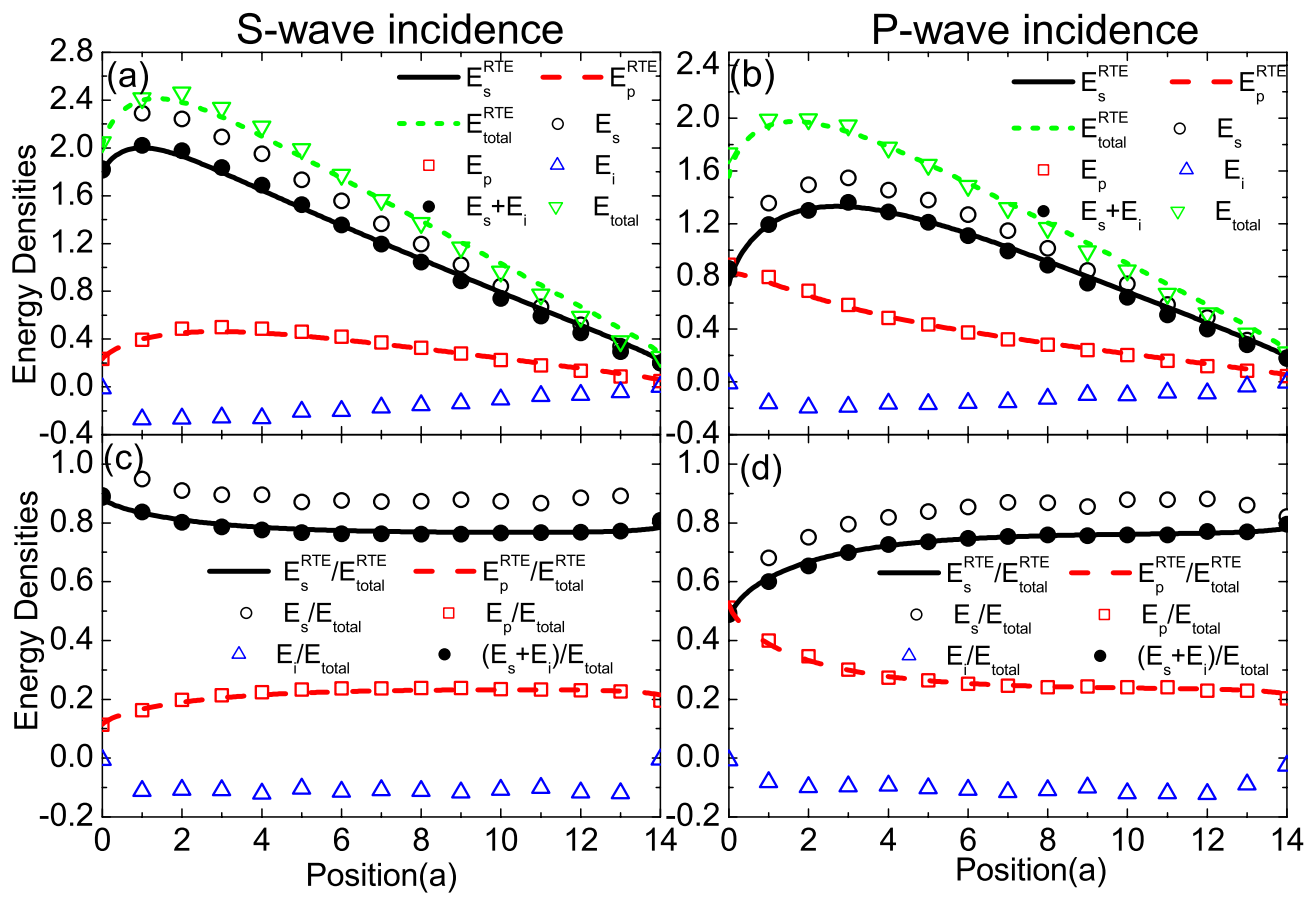

FIG. 3. (Color online) [(a) and (b)] The energy densities inside a slab of random medium for S- and P-wave incidences, respectively. The solid (black), dashed (red), and short-dashed (green) curves correspond to the shear $\left(E_{s}^{R T E}\right)$, compressional $\left(E_{p}^{R T E}\right)$, and total $\left(E_{\text {total }}^{R T E}\right)$, energy densities obtained from RTE, respectively. The corresponding quantities determined by multiple-scattering simulations are plotted in open (black) circles, open (red) squares, and open down (green) triangles. The open up (blue) triangles represent simulated interference energy density $\left\langle E_{i}\right\rangle$ and the solid (black) circles are the simulated $\left\langle E_{s}\right\rangle+\left\langle E_{i}\right\rangle$. [(c) and (d)] Energy percentage for S- and P-wave incidences, respectively. The solid (black) and dashed (red) curves show that the percentage of shear and compressional energy densities occupies the total energy density, i.e., $E_{s}^{R T E} / E_{\text {total }}^{R T E}$ and $E_{p}^{R T E} / E_{\text {total }}^{R T E}$, respectively. The open (black) circles, open (red) squares, open upper (blue) triangles, and solid (black) circles correspond to the results of $\left\langle E_{s}\right\rangle /\left\langle E_{\text {total }}\right\rangle,\left\langle E_{p}\right\rangle /\left\langle E_{\text {total }}\right\rangle,\left\langle E_{i}\right\rangle /\left\langle E_{\text {total }}\right\rangle$, and $\left(\left\langle E_{s}\right\rangle+\left\langle E_{i}\right\rangle\right) /\left\langle E_{\text {total }}\right\rangle$ obtained from the MST method, respectively.

equilibration process. In other words, after some sufficient number of scatterings, the ratios of the shear energy density to the compressional energy density obtained from two different incident waves converge to a single constant number, except for a slight upturn near the output surface. However, their equilibrated ratios are different. The equilibrated ratio obtained from the RTE agrees well with the prediction of the equipartition principle, i.e., $E_{s}^{R T E} / E_{p}^{R T E}=\left(c_{p} / c_{s}\right)^{2} \cong 3.26$, whereas the result obtained from the MST method is $E_{s} / E_{p}$ $\cong 3.7$, which is larger than the prediction of the equipartition principle. This is expected from Fig. 3(a), which shows $\left\langle E_{s}\right\rangle>E_{s}^{R T E}$ due to the presence of a negative interference energy density, $\left\langle E_{i}\right\rangle$. However, if the ratio of $\left(\left\langle E_{s}\right\rangle\right.$ $\left.+\left\langle E_{i}\right\rangle\right) /\left\langle E_{p}\right\rangle$ is plotted, there is an excellent agreement with $E_{s}^{R T E} / E_{p}^{R T E}$, as shown in Fig. 4(b). This is also expected from the results shown in Fig. 3(a).

The slight upturn of the ratio found in Fig. 4 is due to the absence of backward scatterings coming from outside the sample. To show this, we have done the following calculation. We considered a sample of thickness $L=28 a$ and solved the RTE to obtain $E_{s}^{R T E}$ and $E_{p}^{R T E}$ inside the sample. The ratio $E_{s}^{R T E} / E_{p}^{R T E}$ near the middle of the sample has the equilibrated value of 3.26. Then, by using Eq. (13), we calculated, to the first-order scattering, the energy densities at the middle point of the sample contributed by the backward energy current density coming from the left-half of the sample. It was found

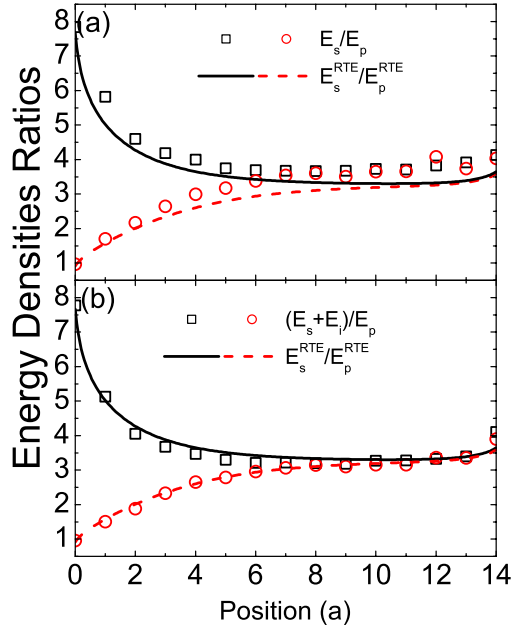

FIG. 4. (Color online) Ratios of energy densities determined from different methods inside the random media. [(a) and (b)] Comparison between the ratios determined from RTE and MST. (a) The solid black and dashed red curves represent $E_{s}^{R T E} / E_{p}^{R T E}$ obtained from RTE for S- and P-wave incidences, respectively. The squares (black) and circles (red) are $\left\langle E_{s}\right\rangle /\left\langle E_{p}\right\rangle$ determined by the MST method for S- and P-wave incidences, respectively. (b) The solid black and dashed red curves are the same as those in (a). The squares (black) and circles (red) are $\left(\left\langle E_{s}\right\rangle+\left\langle E_{i}\right\rangle\right) /\left\langle E_{p}\right\rangle$ determined by the MST method for S- and P-wave incidences, respectively. 
that the ratio of the backward shear energy density to the compressional energy density was 3.12 , which is smaller than the equilibrated value of 3.26. This implies that, relatively, the shear energy density has less contribution from the backward current than the compressional energy density. Since there is no backward current at the sample boundary, the ratio of shear energy density to the compressional energy density will increase accordingly.

To understand the origin of the negative $\left\langle E_{i}\right\rangle$ and the interesting agreement between the sum $\left\langle E_{s}\right\rangle+\left\langle E_{i}\right\rangle$ and $E_{s}^{R T E}$, we normalized various energy densities in each calculation by its total energy density. The results are plotted in Figs. 3(c) and 3(d). It is shown that the functions $\left\langle E_{p}\right\rangle /\left\langle E_{\text {total }}\right\rangle$ and $\left(\left\langle E_{s}\right\rangle\right.$ $\left.+\left\langle E_{i}\right\rangle\right) /\left\langle E_{\text {total }}\right\rangle$ obtained from the MST calculation agree well with $E_{p}^{R T E} / E_{\text {total }}^{R T E}$ and $E_{s}^{R T E} / E_{\text {total }}^{R T E}$ obtained from the RTE, respectively, whereas the ratio of shear energy density to the total energy density obtained from the two methods are different as expected. From these figures, we also find that the interference energy occupies about $10 \%$ of the total energies for both S- and P-wave incidences, which is quite uniform across the sample except near the boundaries. It should be pointed out that the interference energy is generally believed to be averaged to zero inside the bulk random media. ${ }^{10}$ This is true for weak-scattering random media, where the nearfield effects due to the scattering can be ignored and the RTE can be applied. However, in the strong-scattering random medium studied here, both the S- and P-wave wavelengths corresponding to the frequency, $\tilde{f}=2.4$, i.e., $\lambda_{s} \cong 0.4 a$ and $\lambda_{p} \cong 0.7 a$, are comparable to the average separation between two scatterers, i.e., $a$. Thus, the interference energy density in the neighborhood of each scattering constitutes a nonnegligible part of the total energy density and such near-field effects cannot be averaged out after configurational averaging. ${ }^{25}$ To check this point, we redid the calculation by using the same scatterers and frequency, but reduced the number density of the scatterers by a factor $1 / 4$. Our new results are similar to the ones shown in Fig. 3, except that the interference energy now occupies about $5 \%$ of the total energy. This confirms the above explanation that the negative interference energy is due to the near-field effects and is expected to vanish in the weak-scattering limit where the average distance between two neighboring scatterers is much greater than the wavelengths. Since the number density of the scatterers is zero immediately outside our sample, the interference energy density also approaches zero in a distance $a$ within the sample boundary, as can been seen in Figs. 3(c) and 3(d).

To understand better the distribution of the inference energy density in the vicinity of each cylinder, we also calculated various energy density distributions resulting from a plane-wave scattering by a single cylinder. To show these results, we first integrated the energy density, $E\left(\vec{r}^{\prime}\right)$, in an annular ring defined by $r-\frac{d r}{2}<\left|\vec{r}^{\prime}\right|<r+\frac{d r}{2}$ centered at the cylinder and divided by its area, $2 \pi r d r$. The angle-averaged energy density distributions are plotted in Fig. 5 as functions of the distance from the center of the cylinder for both the S-wave incidence [Fig. 5(a)] and the P-wave incidence [Fig. 5(b)], where the solid, dashed, and the dash-dotted curves correspond to the shear, compressional, and interference den-



FIG. 5. (Color online) Various angle-averaged energy density distributions for a plane-wave scattering on a single cylinder. The black solid, red dashed, and blue dash-dotted curves represent shear, compressional, and interference energy densities, respectively. The shear plus interference energy densities are plotted in the green short-dashed curves; (a) for S-wave incidence and (b) for P-wave incidence.

sities, respectively. The short-dashed curves denote the sum of the shear and interference energy densities. Figure 5(a) shows a large negative peak in the interference energy density near the boundary of the cylinder, i.e., $r=0.15$. Its magnitude is about four times of the shear energy density away from the boundary. This large negative peak cannot be averaged to zero by any random environment and is the main source of the negative interference energy density found in Fig. 3. Away from the boundary, the interference energy density is small and exhibits oscillatory behavior, which is expected to be averaged out in a random environment. It is interesting to see that, accompanied by the presence of a large negative peak in the interference energy density, there also exists a large enhancement in the shear energy density by the similar magnitude near the boundary of the cylinder. Thus, the cancellation of two peaks in the sum of these two energy densities gives a smooth short-dashed curve in Fig. 5(a). This explains why the sum of the shear and interference energy densities calculated by the MST method agrees so well with the shear energy density obtained from the RTE as the large negative interference energy density is compensated by the large positive enhancement in the shear energy density. Figure 5(b) shows the results of the P-wave incidence. In this case, there exists a negative interference energy density inside the cylinder. However, its magnitude is more than half of the compressional energy density away from the cylinder. Since the compressional energy density is smaller than the shear energy density by roughly a factor 3.7 , the negative interference energy induced by the P-wave scattering can only make a small contribution to the negative energy density found in Fig. 3. Even so, the existence of the negative interference energy density is also accompanied by a similar enhancement in the shear energy density. 


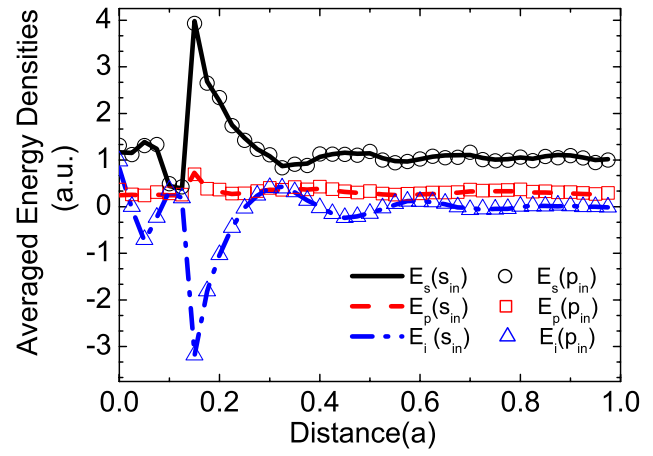

FIG. 6. (Color online) Various equilibrated energy density distributions as functions of distance from the center of a cylinder inside a random medium. The black solid, red dashed, and blue dash-dotted curves represent shear, compressional, and interference energy densities, respectively, for S-wave incidence. The black circles, red squares, and blue triangles correspond to shear, compressional, and interference energy densities, respectively, for $\mathrm{P}$-wave incidence.

It should be pointed out that, strictly speaking, a higher than expected equilibrated ratio of $\left\langle E_{s}\right\rangle /\left\langle E_{p}\right\rangle$ does not necessary implies a violation of the principle of equipartition of wave modes. The wave modes considered in this work are Sand P-polarized waves of the host medium. The presence of scatterers can strongly alter the mode structures as well as the density of states in the vicinity of a scatterer. The altered eigenstates in general are mixtures of S- and P-polarized waves, which give rise to a nonzero interference energy density. Although there is no simple and direct method to demonstrate the principle of equipartition in a strongly scattered medium, we try to show it indirectly by calculating various averaged energy density distributions close to a scatterer located well inside a sample using both S- and P-wave incidences. If equipartition principle holds, these distributions should be independent of the polarization of the source.

To study this, we increased the lattice constant of the ordered phononic crystal from $a$ to $2 a$. The random samples studied here have a width of $W=100 a$, or equivalently, 50 layers, and a length of $L=40 a$, i.e., 20 layers. Therefore, the total number of scatterers in each sample is 1000 . In the randomization procedure, for the convenience of this study, we imposed a condition that any two cylinders should be at least $1 a+2 r_{s}$ apart. To calculate the energy density distributions near a scatterer, we picked up 25 cylinders located well inside the sample and away from the incident wave so that the energy equilibration has already been established. We then calculated the angle-averaged energy distributions for the shear, compressional, and interference terms as functions of distance away from the center of each cylinder. The calculations were repeated for 200 different configurations. The averaged results of 5000 different local configurations of the scatterers are shown in Fig. 6. The black solid, red dashed, and blue dash-dotted curves represent shear, compressional, and interference energy density distributions, respectively, for the S-wave incidence. The corresponding results of a $\mathrm{P}$-wave incidence are shown by the black circles, red squares, and blue triangles, respectively. It is clearly seen that these distributions are independent of the source polar- ization. It is also seen that away from the scatterer, the interference energy densities die off quickly and the ratio of the shear and compressional energy densities approaches to that of the host medium, i.e., $\left(c_{p} / c_{s}\right)^{2} \cong 3.26$. These results demonstrate that the principle of equipartition holds in general and a higher than expected equilibrated ratio of $\left\langle E_{s}\right\rangle /\left\langle E_{p}\right\rangle$ found in this work is a result of modification of mode structures near the scatterers.

\section{CROSSOVER FROM BALLISTIC TO DIFFUSIVE BEHAVIOR}

By using the MST method, we also studied the field distributions of the transmitted waves in thin samples before energy equilibration occurs. These results were analyzed by the RPS model, ${ }^{12}$ from which we also observed crossover behavior from the ballistic to diffusive regions.

As discussed in Sec. II, for an S-polarized (or P-polarized) plane wave propagating along the $x$ direction, the displacement field $\mathbf{u}$ is along the $y$ or $x$ direction. The transmitted displacement field along the $y$ or $x$ direction can be decomposed into two terms:

$$
u_{x, y}=\left\langle u_{x, y}\right\rangle+\delta u_{x, y} .
$$

The first term $\left\langle u_{x, y}\right\rangle$ represents the coherent part of the transmitted waves, where the angular brackets represent the ensemble average over different disorder realizations, and the second term $\delta u_{x, y}$ represents the residue part of the waves. In this section, we present a statistical analysis of both $u_{x, y}$ and $\delta u_{x, y}$. To do so, we express the complex field $u_{x, y}$ as $u_{y}$ $=u_{y 0} e^{i \phi}$ for the S-wave incidence and $u_{x}=u_{x 0} e^{i \phi}$ for the $\mathrm{P}$-wave incidence, where $u_{x 0, y 0}$ is the amplitude and $\phi$ denotes the phase.

In the RPS model, a normalized amplitude, $A=u_{x 0, y 0} / \sigma$, is used, where $2 \sigma=\left\langle\left|u_{x, y}\right|{ }^{2}\right\rangle-\left|\left\langle u_{x, y}\right\rangle\right|^{2}$, and the amount of the coherent component of the total field is represented by a parameter, $k=\left|\left\langle u_{x, y}\right\rangle\right| / \sigma$. The RPS model gives the following distribution functions for the amplitude and phase: ${ }^{12}$

$$
P(A, k)=A \exp \left(-\frac{A^{2}+k^{2}}{2}\right) I_{0}(A k),
$$

and

$$
\begin{aligned}
P(\phi, k)= & \frac{1}{2 \pi} \exp \left(-k^{2} / 2\right)+\frac{k \cos \phi}{\sqrt{2 \pi}} \\
& \times \exp \left(-\frac{(k \sin \phi)^{2}}{2}\right) \operatorname{erf}(k \cos \phi),
\end{aligned}
$$

where $I_{0}(x)$ is a modified Bessel function of the first kind of order zero, and $\operatorname{erf}(x)$ is an error function.

We first consider a thin sample with thickness $L=2 a$. The samples used here again have a width $W=50 a$ and number density $1 / a^{2}$. Both S- and P-wave incidences are considered. For each configuration, we collected 101 data points of the transmitted field $\mathbf{u}$ along the $y$ axis in a range from $-10 a$ to $10 a$ at a distance $2 a$ away from the output surface. Results of 200 random configurations were obtained. From this en- 

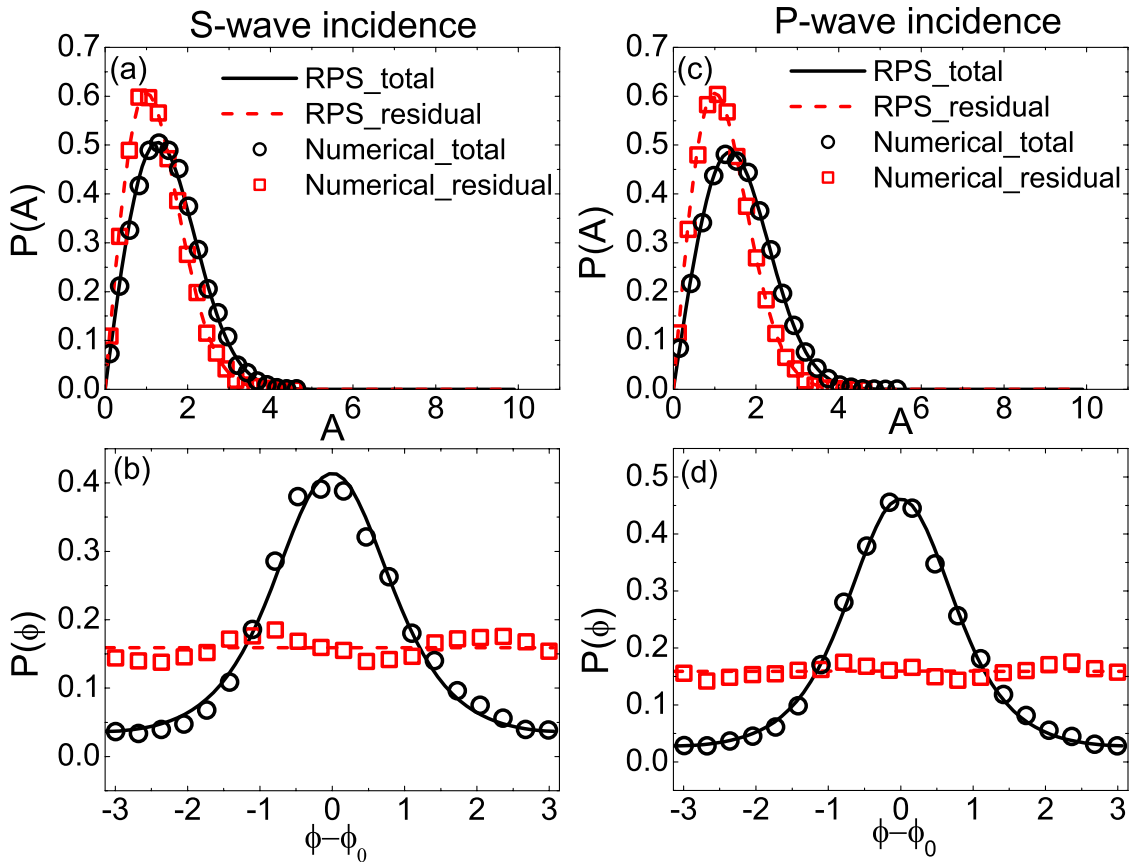

FIG. 7. (Color online) Normalized amplitude (a) and phase (b) distribution in the sample with a thickness of $2 a$ for the S-wave incidence. The open black circles and open red squares are the total and residual field distributions calculated by the MST method, respectively. The solid (black) lines and dashed (red) lines are the theoretical predictions of the total and residual fields made by the RPS model, respectively. (c) and (d) exhibit the same quantities as (a) and (b), correspondingly, but the incident wave is a compressional wave.

semble of $101 \times 200$ data points, we studied the statistical distributions of both the normalized amplitude $A$ and the phase $\phi$. The results of the S-wave incidence are shown in Figs. 7(a) and 7(b), where the open circles represent the amplitude and phase distributions of the total field and the open squares are used for the residual field. To compare the results with the RPS model, we also plot the results of Eqs. (16) and (17) in Figs. 7(a) and 7(b), where the solid lines represent the amplitude and phase distributions of the total field and the dashed lines are used for the residual field. Here, we have used the value of $k=0.95$ obtained from the numerical simulations for the total field, and $k=0$ is used for the residual field. From Figs. 7(a) and 7(b), we find excellent agreements between the distributions obtained from the RPS model and numerical simulations.
Similar results are plotted in Figs. 7(c) and 7(d) for the case of $\mathrm{P}$-wave incidence. In this case, numerical simulations give $k=1.09$ for the total field. Excellent agreement between the RPS model and numerical simulations are clearly seen in this case. Since the sample thickness is only $2 a$, which is between one to two mean free paths for both $\mathrm{S}$ and $\mathrm{P}$ waves, there still exists a significant amount of the coherent component in the transmitted waves. However, the amplitude distribution of the residual field is always of the Rayleigh type as can be seen clearly from Figs. 7(a) and 7(c).

Next, we increase the sample thickness to $L=8 a$, which is slightly thinner than the thickness at which energy equilibration is established. The amplitude and field distributions are plotted in Fig. 8 for both S- and P-wave incidences. Figure 8 exhibits good agreement between the RPS result and the
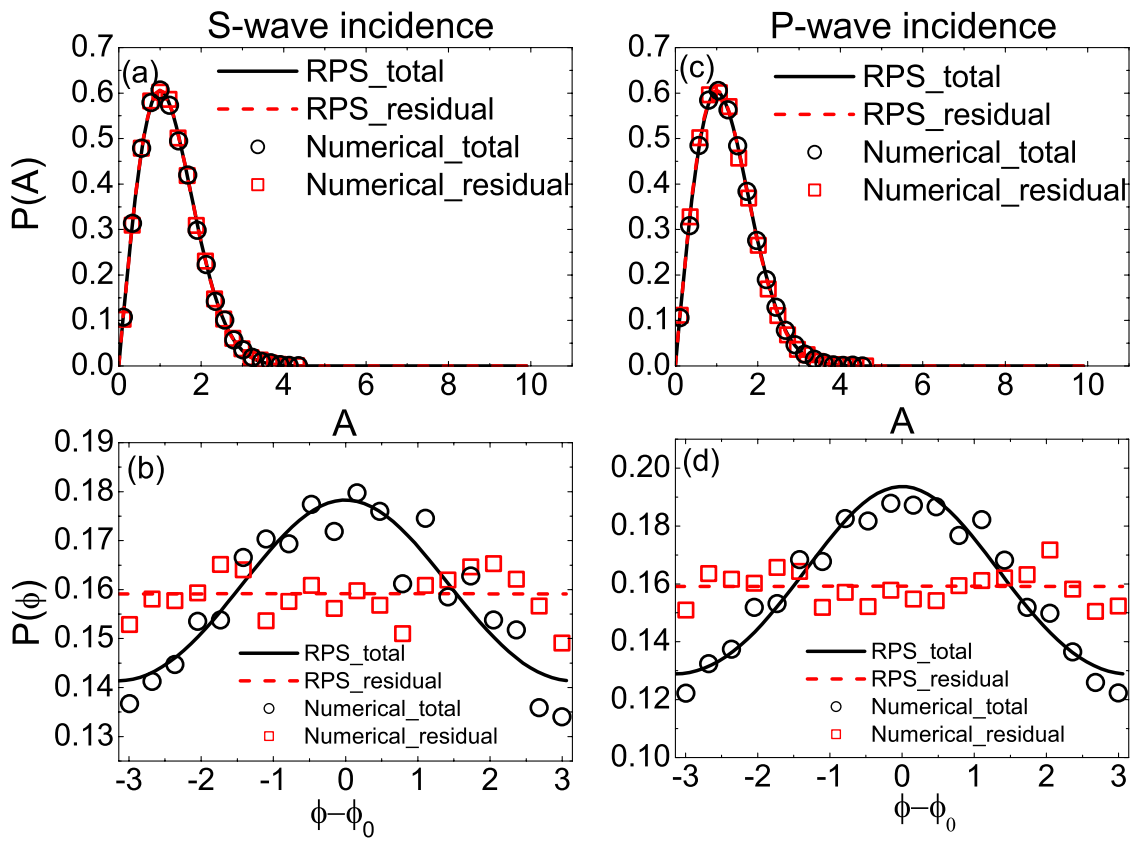

FIG. 8. (Color online) Normalized amplitude (a) and phase (b) distribution in the sample with a thickness of $8 a$ for the S-wave incidence. The open black circles and open red squares are the total and residual field distribution calculated by the MST method, respectively. The solid (black) lines and dashed (red) lines are the theoretical predictions of the total and residual fields made by the RPS model, respectively. (c) and (d) exhibit the same quantities as (a) and (b), correspondingly, but the incident wave is a compressional wave. 
simulated data. In this case, the simulation results give $k$ $=0.09$ and 0.16 for $\mathrm{S}$ - and P-wave incidences, respectively. Since the value of $k$ is about an order of magnitude smaller than the case of the $L=2 a$ sample, a much smaller coherent component is found in this thicker sample. In this case, the amplitude distribution of the total field becomes indiscernible to that of the residual field, as shown in Figs. 8(a) and 8(c). However, a slight deviation from complete randomization in the phase distribution of the total field can still be seen in Figs. 8(b) and 8(d). Thus, the establishment of energy equilibration does not necessarily imply a complete randomization of the incident wave. This observation is consist with a previous study on the dynamics of energy equilibration of elastic waves in the seismic coda. ${ }^{26}$ Figures 7 and 8 also exhibit crossover behavior from ballistic transport to diffusive transport in strongly scattered elastic media.

\section{CONCLUSIONS}

By using the MST method in conjunction with the SMCG method, we have numerically studied elastic wave propagation through strongly scattered random media. The spatial distributions of the calculated compressional and total energy densities agree well with those obtained from the RTE. However, a negative interference energy is found inside the sample. By studying various energy density distributions arising from the plane-wave scattering of a single cylinder, we found that the negative interference energy density occurs close to the boundary of the cylinder, and, therefore, cannot be averaged to zero. Accompanying the negative interference energy density is the enhancement of the shear energy density by a similar magnitude. Thus, the sum of the two energy densities coincides with the shear energy density calculated by the radiative transport equation. Both results show clearly an energy equilibration process. The equilibrated ratio of the shear to compressional energy density in the RTE calculations agrees excellently with the principle of equipartition of two wave modes. However, a higher value is found in the multiple-scattering calculations due to the presence of the negative interference energy density. We have also studied the statistical properties of transmitted waves through thin samples by separating the transmitted fields into coherent and residual parts. It has been found that the field and phase distributions of both residual and total fields can be well described by the RPS model and they exhibit crossover behavior from ballistic to diffusive wave propagation in strongly scattered random elastic media.

\section{ACKNOWLEDGMENT}

This work was supported by Hong Kong RGC Grant No. 604703.
${ }^{1} \mathrm{P}$. Sheng, Introduction to Wave Scattering, Localization and Mesoscopic Phenomena (Academic, New York, 1995).

${ }^{2}$ H. Sato and M. Fehler, Seismic Wave Propagation and Scattering in the Heterogeneous Earth (Springer, New York, 1998).

${ }^{3}$ S. Hirsekorn, J. Acoust. Soc. Am. 83, 1231 (1987).

${ }^{4}$ R. L. Weaver, J. Mech. Phys. Solids 38, 55 (1990).

${ }^{5}$ R. L. Weaver, J. Acoust. Soc. Am. 71, 1608 (1982).

${ }^{6}$ G. C. Papanicolaou, L. V. Ryzhik, and J. B. Keller, Bull. Seismol. Soc. Am. 86, 1107 (1996).

${ }^{7}$ J. A. Turner, Bull. Seismol. Soc. Am. 88, 276 (1998).

${ }^{8}$ Nicolas P. Trégourès and Bart A. van Tiggelen, Waves Random Media 12, 21 (2002).

${ }^{9}$ For example, E. N. Economou, Green's Functions in Quantum Physics (Springer-Verlag, New York, 1979).

${ }^{10}$ R. Hennino, N. Tregoures, N. M. Shapiro, L. Margerin, M. Campillo, B. A. van Tiggelen, and R. L. Weaver, Phys. Rev. Lett. 86, 3447 (2001).

${ }^{11}$ C. H. Chan and L. Tsang, Microwave Opt. Technol. Lett. 8, 114 (1995).

${ }^{12}$ A. A. Chabanov and A. Z. Genack, Phys. Rev. E 56, R1338 (1997).

${ }^{13}$ A. Garcia-Martin, J. J. Saenz, and M. Nieto-Vesperinas, Phys. Rev. Lett. 84, 3578 (2000).
${ }^{14}$ X. D. Zhang and Z. Q. Zhang, Phys. Rev. B 65, 245115 (2002).

${ }^{15} \mathrm{~K}$. Younge, C. Christenson, A. Bohara, J. Crnkovic, and P. Saulnier, Am. J. Phys. 72, 1247 (2004).

${ }^{16}$ M. S. Kushwaha, P. Halevi, G. L. Martínez, L. Dobrzynski, and B. Djafari-Rouhani, Phys. Rev. B 49, 2313 (1994).

${ }^{17}$ Y. Wu, Y. Lai, and Z. Q. Zhang, Phys. Rev. B 76, 205313 (2007).

${ }^{18}$ L. M. Li and Z. Q. Zhang, Phys. Rev. B 58, 9587 (1998).

${ }^{19}$ Y. Lai and Z. Q. Zhang, Appl. Phys. Lett. 83, 3900 (2003).

${ }^{20}$ J. Mei, Z. Liu, J. Shi, and D. Tian, Phys. Rev. B 67, 245107 (2003).

${ }^{21} \mathrm{~S}$. Yousef, Iterative Methods for Sparse Linear Systems (PWS, Boston, 1996).

${ }^{22}$ P. M. Morse and H. Feshbach, Methods of Theoretical Physics (McGraw-Hill, New York, 1953).

${ }^{23}$ J. O. Vasseur, P. A. Deymier, B. Chenni, B. Djafari-Rouhani, L. Dobrzynski, and D. Prevost, Phys. Rev. Lett. 86, 3012 (2001).

${ }^{24}$ L. V. Ryzhik, G. C. Papanicolaou, and J. B. Keller, Wave Motion 24, 327 (1996).

${ }^{25}$ Thorne Lay and Terry C. Wallace, Modern Global Seismology (Academic, San Diego, 1995).

${ }^{26}$ L. Margerin, B. van Tiggelen, and M. Campillo, Bull. Seismol. Soc. Am. 91, 624 (2001). 